\title{
The ZEUS measurement of charged current DIS cross sections with a longitudinally polarised positron beam
}

\author{
Amita Raval* \\ $D E S Y / C E R N$ \\ E-mail: amita.ravalecern.ch

\section{Katie Oliver} \\ University of Oxford \\ E-mail: k.oliver1@physics.ox.ac.uk
}

\begin{abstract}
The ZEUS measurements of charged current deep inelastic scattering cross sections in $e^{+} p$ collisions with a longitudinally polarised positron beam are presented. The data sample used has an integrated luminosity of $132 \mathrm{pb}^{-1}$ at a centre-of-mass energy of $318 \mathrm{GeV}$. The total cross section is presented at positive and negative values of the longitudinal polarisation of the positron beams. The single-differential cross-sections $d \sigma / d Q^{2}, d \sigma / d x$ and $d \sigma / d y$ have been measured for $Q^{2}>200 \mathrm{GeV}^{2}$ but are not presented here. The reduced cross-section $\tilde{\sigma}$ is presented in the kinematic range $200<Q^{2}<60000 \mathrm{GeV}^{2}$ and $0.006<x<0.562$. A lower limit on the mass of a hypothetical $W$ boson which couples to right-handed particles has been determined.
\end{abstract}

XVIII International Workshop on Deep-Inelastic Scattering and Related Subjects April 19 -23, 2010

Convitto della Calza, Firenze, Italy

\footnotetext{
${ }^{*}$ Speaker.
} 


\section{Introduction}

Both an improved understanding of QCD and precise measurements of the proton parton density functions (PDFs) have been made possible in part by the study of charged current (CC) deep inelastic scattering (DIS) processes at the $e p$ collider HERA. The CC process is mediated by the exchange of a $W^{ \pm}$boson. The events may be described by three invariant variables: $Q^{2}$, the virtuality of the $W^{ \pm} ; x$, the Bjorken scaling variable; and $y$, the inelasticity. These are related by $Q^{2}=s x y$ where $s$ is the centre-of-mass energy squared. The kinematic variables were estimated using the Jacquet-Blondel method [1].

In 2002, HERA was upgraded to provide longitudinal polarisation $\left(P_{e}\right)$ of the lepton beam. This has made it possible to test the chiral nature of the Standard Model since the total CC cross section is expected to go to zero for a fully left-handed incoming positron beam, $\sigma\left(P_{e}\right)=(1+$ $\left.P_{e}\right) \sigma\left(P_{e}=0\right)$. The longitudinal polarisation of the positron beam is defined as

$$
P_{e}=\frac{N_{R}-N_{L}}{N_{R}+N_{L}}
$$

where $N_{R}$ and $N_{L}$ are the numbers of right- and left-handed positrons in the beam.

This paper reviews the recent ZEUS measurements of the inclusive CC cross sections at high $Q^{2}$ using data collected during 2006-2007.

\section{Data Selection}

The $2006 / 2007 e^{+} p$ dataset was split into two polarisation subsets: a positive polarisation sample with mean polarisation of $+33 \%$ and an integrated luminosity of $75.8 \mathrm{pb}^{-1}$ and a negative polarisation sample with mean polarisation of $-36 \%$ and an integrated luminosity of $56.0 \mathrm{pb}^{-1}$. Thus the total integrated luminosity of the sample is $132 \mathrm{pb}^{-1}$ with a residual polarisation of $+3.4 \%$. The uncertainty on the measurement of the luminosity is $2.6 \%$. The uncertainties on the measurements of polarisation are $4.0 \%$ and $3.7 \%$ for the negative and positive polarisation samples respectively.

Charged current DIS candidate events were selected by requiring all events to have large missing transverse momentum, $P_{T, \text { miss }}$. The missing transverse momentum arises because the final-state neutrino is undetected and carries off some of the transverse momentum. The kinematic region was restricted to $Q^{2}>200 \mathrm{GeV}^{2}$ and $y<0.9$ where the backgrounds were manageable and the detector response was well simulated.

Backgrounds to the $\mathrm{CC}$ process were reduced using a combination of cuts. Non-ep backgrounds were greatly reduced by making cuts on the quality of tracks, in particular, the requirement of an $e p$ interaction vertex. Cosmic $\mu$ rejection was achieved by comparing the fraction of energy deposited in the electromagnetic and hadronic calorimeters. Photoproduction and beam gas events were rejected with jet shape cuts since $\mathrm{CC}$ events have much more collimated jets. Neutral current events were rejected using cuts on electron variables. A total of 2327 data events satisfied all criteria in the positive-polarisation sample and 821 events in the negative-polarisation sample. The largest backgrounds were photoproduction ( $0.6 \%$ of the final sample), single- $W$ production $(0.3 \%)$ and di-leptons $(0.2 \%)$. The contamination from NC events was estimated to be very small and non-e $p$ backgrounds were negligible. In bins of the single- and reduced cross-section bins, the 
background contamination was typically less than $1.5 \%$. Figure 1 compares the distributions of data events entering the final $\mathrm{CC}$ sample with the $\mathrm{MC}$ expectation for the sum of the $\mathrm{CC}$ signal and $e p$ background events.

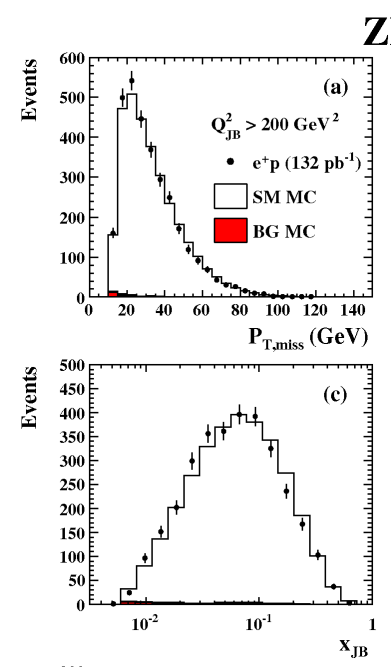

ZEUS

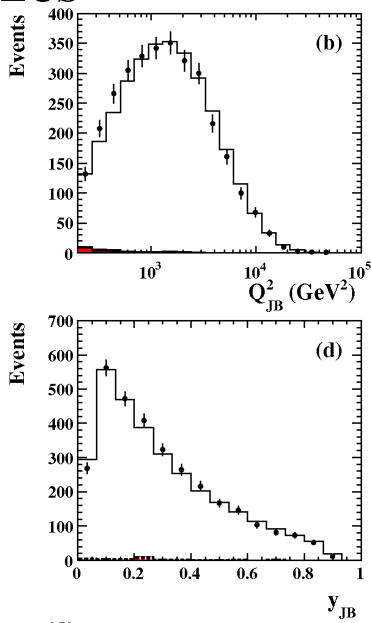

Figure 1: Comparison of the total $e^{+} p \mathrm{CC}$ data sample (points) with MC. The open (filled) histograms represent the signal (background) MC. The distributions of (a) $P_{T, \text { miss }}$, (b) $Q_{\mathrm{JB}}^{2}$, (c) $x_{\mathrm{JB}}$ and (d) $y_{\mathrm{JB}}$ are shown.

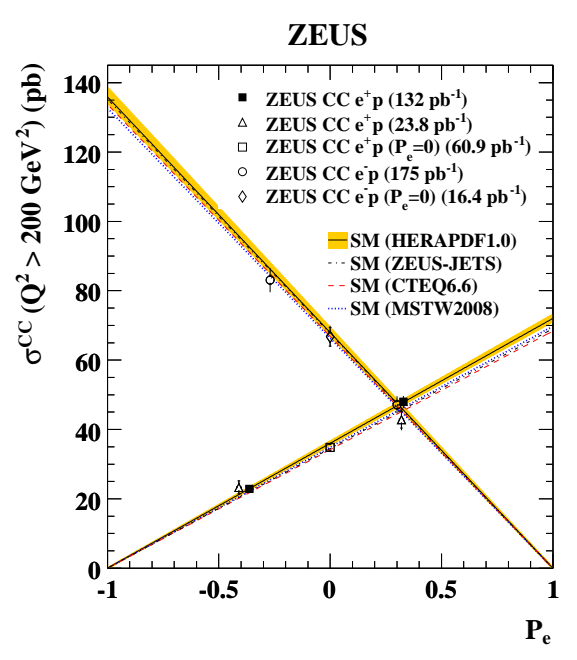

Figure 2: The total cross sections for $e^{+} p$ and $e^{-} p$ CC DIS as a function of lepton beam polarisation. The SM predictions obtained with HERAPDF1.0 (with uncertainty shown as shaded band), ZEUS-JETS, CTEQ6.6 and MSTW2008 PDFs are shown.

\section{Total Cross Sections}

The $e^{+} p$ CC DIS total cross section in the kinematic region $Q^{2}>200 \mathrm{GeV}^{2}$, corrected to Born level in the electroweak interaction, was measured to be:

$$
\begin{aligned}
& \left.\sigma^{\mathrm{CC}}\left(P_{e}=-0.36\right)=22.9 \pm 0.82 \text { (stat.) } \pm 0.60 \text { (lumi. }\right) \pm 0.40 \text { (syst.) } \mathrm{pb} \\
& \left.\sigma^{\mathrm{CC}}\left(P_{e}=+0.33\right)=48.0 \pm 1.01 \text { (stat.) } \pm 1.25 \text { (lumi. }\right) \pm 0.77 \text { (syst.) pb }
\end{aligned}
$$

The total cross section is shown as a function of the longitudinal polarisation of the lepton beam in Fig. 目along with previous ZEUS measurements from both $e^{-} p$ and $e^{+} p$ data [2, 3, 4, 5]. The SM predicts the CC $e^{+} p$ DIS cross section to be zero for fully left-handed positron beams, thus a non-zero cross section at $P_{e}=-1$ might point to the existence of a $W$ boson which couples to right-handed particles $\left(W_{R}\right)$ 6 行. A linear function was fit to the total cross section in 8 bins of polarisation and extrapolated to $P_{e}=-1$. The upper limit on the cross section was converted to a lower limit on the mass of $W_{R}\left(M_{W_{R}}\right)$. It was assumed that the final-state neutrino is light and that the coupling strength and propagator dependence on the mass of the boson are the same as in SM $\mathrm{CC}$ interactions. The limits obtained are:

$$
\sigma^{\mathrm{CC}}\left(P_{e}=-1\right)<2.9 \mathrm{pb} \text { at } 95 \% \mathrm{CL}
$$




$$
M_{W_{R}}>198 \mathrm{GeV} \text { at } 95 \% \mathrm{CL}
$$

This limit is for a space-like $W$ and so may be viewed as complementary to the limits obtained from direct searches [8] where the $W$ boson is time-like.

\section{Reduced Cross Sections}

The reduced cross-section $\tilde{\sigma}$ for $\mathrm{CC} e^{+} p$ scattering is given by:

$$
\tilde{\sigma}=\left(1+P_{e}\right)[\bar{u}+\bar{c}+(1-y) 2(d+s)]
$$

where, for example, the PDF $d\left(x, Q^{2}\right)$ gives the number density of down quarks with momentumfraction $x$ at a given $Q^{2}$.

The reduced cross section is measured in the kinematic range $200<Q^{2}<60000 \mathrm{GeV}^{2}$ and $0.006<x<0.562$ and is shown as a function of $x$ at fixed values of $Q^{2}$ in Figure 3 (left). The predictions of the SM evaluated using the HERAPDF1.0 [9], ZEUS-JETS [10], CTEQ6.6 [11] and MSTW2008 [12] PDFs give a good description of the data. The contributions from the HERAPDF1.0 PDF combinations $(d+s)$ and $(\bar{u}+\bar{c})$ are shown separately. The helicity structure of CC interactions can be illustrated by plotting the reduced cross section versus $(1-y)^{2}$ in bins of $x$ as shown in Fig. 3 (right). In the region of approximate scaling, i.e. $x \approx 0.1$, this yields a straight line. At leading order in QCD, the intercept of this line gives the $(\bar{u}+\bar{c})$ contribution, while the slope gives the $(d+s)$ contribution. Measurement of the reduced cross sections provides sensitivity to the $d$ valence quark PDF. These measurements therefore have the potential to further constrain PDFs.

\section{Summary}

In this paper, the total, single-differential and reduced charged current $e^{+} p$ DIS cross sections have been presented. The measurements were based on a data set with an integrated luminosity of $132 \mathrm{pb}^{-1}$ taken in $2006 / 2007$ by the ZEUS detector at the HERA collider. The Standard Model describes the data well. The results of this analysis should help to constrain the $d$ valence quark and sea PDFs, which are important inputs for PDFs at the LHC.

\section{References}

[1] F. Jacquet and A. Blondel, Detection of the Charged Current Event-Method II, Proceedings of the Study for an ep Facility for Europe, U. Amaldi (ed.) p.391. Hamburg, Germany.

[2] ZEUS Coll., S. Chekanov et al., Measurement of High- $Q^{2}$ Charged Current Cross Sections in $e^{-} p$ Deep Inelastic Scattering at HERA, Phys. Lett B 539 , 197 (2002) [hep-ex/0205091]. Erratum in Phys. Lett. B 552, 308 (2003).

[3] ZEUS Coll., S. Chekanov et al., Measurement of High $Q^{2}$ Charged Current Cross-Sections in $e^{+} p$ Deep Inelastic Scattering at HERA, Eur. Phys. J C 32, 1 (2003) [hep-ex/ 0307043 ]

[4] ZEUS Coll., S. Chekanov et al., Measurement of High- $Q^{2}$ Deep Inelastic Scattering Cross Sections with a Longitudinally Polarised Positron Beam at HERA, Phys. Lett B 63728 (2006)

[hep-ex/0602026] 


\section{ZEUS}

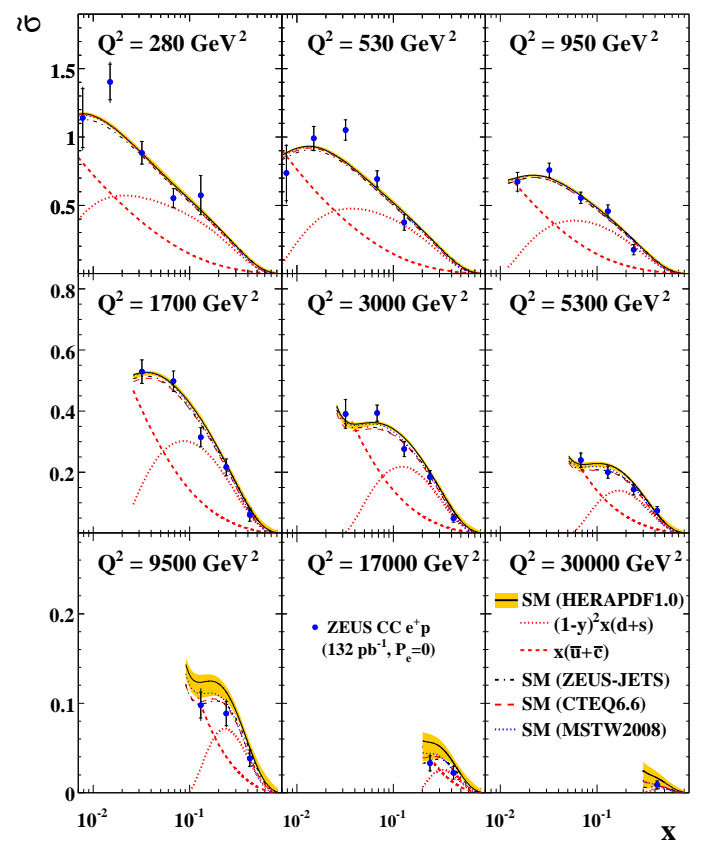

ZEUS

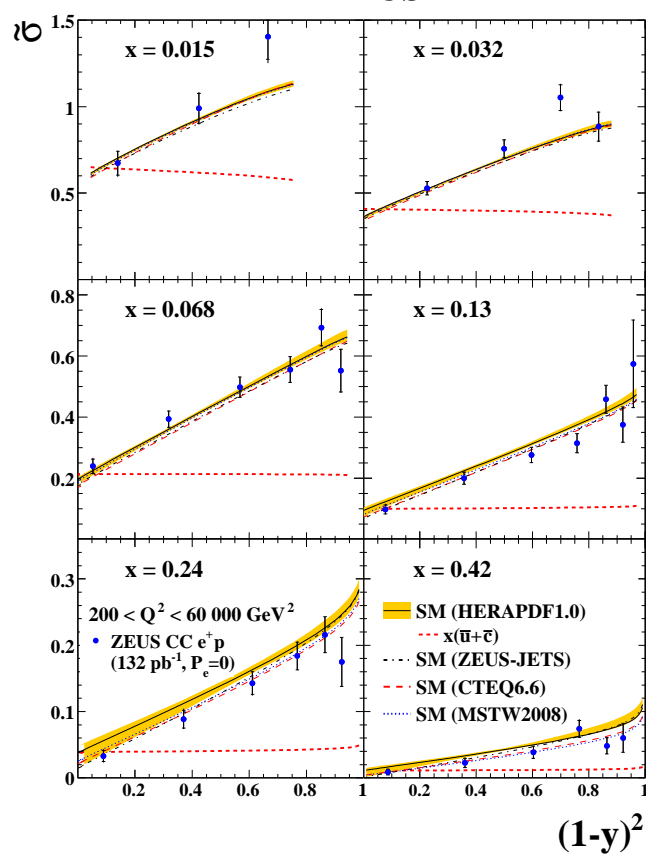

Figure 3: The measured $e^{+} p$ CC DIS reduced cross section plotted as a function of $x$ for fixed $Q^{2}$ (left) and as a function of $(1-y)^{2}$ for fixed $x$ (right). The circles represent the data points and the curves show the predictions of the SM evaluated using the HERAPDF1.0, ZEUS-JETS, CTEQ6.6 and MSTW2008 PDFs. The dashed lines show the contributions of the PDF combination $x(\bar{u}+\bar{c})$ and $(1-y)^{2} x(d+s)$ (left only). The shaded band shows the total uncertainty from the HERAPDF1.0 PDFs.

[5] ZEUS Coll., S. Chekanov et al., Measurement of charged current deep inelastic scattering cross sections with a longitudinally polarised electron beam at HERA, Eur. Phys. J. C 61, 223 (2009) [arXiv: 0812.4620]

[6] G. Senjanovic and R.N Mohapatra Exact Left-Right Symmetry and Spontaneous Violation of Parity, Phys. Rev. D12 1502 (1975)

[7] R.N. Mohapatra and J.C. Pati "Natural" left-right symmetry, Phys. Rev. D11, 2558-2561 (1975)

[8] Particle Data Group, W.-M. Yao et al., Review of Particle Physics, J. Phys G 33, 1 (2006)

[9] H1 and ZEUS Colls., F.D Aaron et al. Combined Measurement and QCD Analysis of the Inclusive ep Scattering Cross Sections at HERA, JHEP 01, 109 (2010) [hep-ex/ 0911.0884 ].

[10] ZEUS Coll., S. Chekanov et al., An NLO QCD Analysis of Inclusive Cross-Section and Jet-Production Data from the ZEUS Experiment, Eur. Phys. J. C 42, 1 (2005) [hep-ph/0503274].

[11] CTEQ Coll., P.M. Nadolsky et al., Implications of CTEQ global analysis for collider observables, Phys. Rev. D 78, 013004 (2008) [hep-ph/ 0802 . 0007].

[12] A.D Martin, W.J Stirling, R.S Thorne and G. Watt, Parton distributions for the LHC, Eur. Phys. J. C63, 189-285 (2009) [hep-ph/ 0901 . 0002] 